\title{
Neurology or rehabilitation medicine?
}

\author{
D L McLellan
}

\begin{abstract}
Rehabilitation is a process of active change by which a person who is disabled acquires and uses the knowledge and skills necessary for optimal physical, psychological and social function. Rehabilitation medicine is now established in Britain as a specialty concerned primarily with three groups: 1) those with multiple disability; 2) disabled people undergoing personal or social transitions, for example, school leavers; and 3) those with disabilities requiring complex technical or medical solutions. Rehabilitation medicine is distinguished from traditional clinical neurology by its emphasis on teamwork and on the analysis and reduction of disability rather than the diagnosis and treatment of impairment. Both neurology and rehabilitation medicine are dwarfed by the size of the problems they are expected to overcome and there is no justification for competition between the two specialties. The training of neurologists requires fundamental changes if they are to be equipped to assist rehabilitation effectively and contribute to the scientific development of the subject.
\end{abstract}

There is little point in trying to draw a sharp dividing line between clinical neurology and rehabilitation medicine. Both are living and evolving specialties. The crucial issue posed by the title of this paper is how best to meet the needs of people with neurological disabilities. The handicap they experience is the result of their own abilities and disabilities, the barriers posed by their physical environment and by the behavioural reactions of other people. People with disabilities have a right to expect these requirements to be met, and it is therefore essential that services created to help them use resources fairly do not redefine their needs to suit the convenience of those who want to provide such a service.

\section{Rehabilitation medicine}

The objective of rehabilitation medicine is the reduction and prevention of disability and handicap arising from physical and psychological impairments.

In the United Kingdom, rehabilitation medicine was first established as an independent specialty in Scotland in $1972^{1}$ and in England and Wales as recently as 1989. In 1990 the Royal College of Physicians' Committee on Disability issued a discussion paper about the specialty ${ }^{2}$ which emphasised that the core of the direct clinical work carried out by consultants in rehabilitation medicine concerns three groups of patients:

1) Those with multiple disability, especially head injury, multiple sclerosis, stroke, cerebral palsy and organic amnesic syndromes, whose families need training and supporting and whose level of function can be improved or maintained by a rehabilitation medicine service.

2) Those with stabilised or progressive disabilities undergoing major personal or social transitions, that is, disabled school leavers, those whose employment is seriously at risk, those having to re-establish themselves with reduced support following separation or bereavement, and those acquiring new problems with advancing age.

3) Those whose disabilities require complex techical, medical or surgical solutions such as amputees, those requiring special seating or orthoses, and those requiring coordination of treatment provided by a number of different specialties such as orthopaedic surgery, neurology, urology and ophthalmology, as in the case of spina bifida with hydrocephalus.

\section{Specific responsibilities of a consultant in}

\section{rehabilitation medicine}

The Disability Committee's document enumerated the responsibilities of a consultant in rehabilitation medicine as follows:

1) Ensuring that an accurate diagnosis is made of the cause of the disability, with review where the disability changes.

2) Establishing a prognosis and explaining its implications to the patient and relatives, and reviewing it if necessary.

3) Establishing the mechanism and assessing the severity of handicap and assisting the disabled person and their family to acquire the knowledge, skills and attitudes necessary to minimise handicaps.

4) Establishment of an integrated plan for the management of disabling disease in individual patients, taking into account its impact upon the patient and the family, and negotiating agreement with this plan with the patient, family and the other professions involved.

5) Establishment and personal leadership of an inter-disciplinary rehabilitation team.

6) Ensuring that all appropriate medical measures have been carried out:

a) To relieve symptoms (for example, treatment of depression or pain).
Research Unit, University of Southampton, Southampton, UK D L McLellan 
b) To maintain or improve function (such as, motor point blocks for spasticity, provision of appropriate orthosis or wheelchair, performance of regular exercises).

c) To prevent deformity (for example, physical therapy, orthosis or surgery).

d) To prevent deterioration of the underlying conditions (such as, eradicating chronic infection in multiple sclerosis, ensuring that relatives are helped to prevent the deterioration in behaviour that can occur after head injury, ensuring that diabetes and smoking are controlled following an amputation).

7) Liaising with other health, community, statutory and voluntary services to ensure the management plan is followed successfully and auditing the outcome.

8) Assisting with the planning and development of health and health-related services for minimising handicap caused by disease or injury.

9) Educating students, junior staff and consultant colleagues in the principles and results of rehabilitation medicine.

10) Undertaking teaching and research in the field of rehabilitation medicine.

\section{Rehabilitation}

Rehabilitation is best defined as a process of active change by which a disabled person acquires and uses the knowledge and skills required for optimal physical, psychological and social function. ${ }^{3}$ This particular process needs information, the opportunity to discuss problems and the provision of informed advice. It may also call for support of a practical or psychological kind. This support is often needed for the principal carer, if there is one, as well as for the person with the disability. This information, advice and support needs to be authoritative, accurate, comprehensive and available. The acquisition of skills is likely to require therapy of various kinds and sometimes formal training and the medical imperatives of the condition need to be clearly identified and incorporated into the overall plan.

To do this it is essential for the service to be provided in the locality where the person is living because the practicalities of the plan will be determined by what the local objectives are and what resources are available there. The plan has to be negotiated rather than imposed. A named individual has to take a continuing responsibility for monitoring it and sticking with the problems until they have been solved, rather than simply handing them on to some other facility.

Given that there is a medical service in every health district, it is not only people with neurological disease that expect to benefit from the presence of rehabilitation medicine. Other consultants, junior medical staff and general practitioners have the right to expect the kind of contribution to their own general education that we all get from specialists in other fields with whom we work. In this way, people with disabilities in other specialties including neurology should gradually get a better stan- dard of service, with earlier and more appropriate referrals to the relevant rehabilitation team. The other professions involved in rehabilitation will expect, and increasingly in the re-organised National Health Service will depend upon, support from their consultant to obtain the resources to meet their own needs for continuing education and service development.

\section{The practice of rehabilitation}

The practice of rehabilitation, for example, as a professional activity, "includes all means aimed at reducing the impact of disabling and handicapping conditions and at enabling disabled people to achieve optimal social reintegration" (World Health Organisation). It is obvious that this practice must embrace many factors quite distinct from medicine such as vocational training, social skills, personal support (including finance) and housing. Many of the factors that prevent people with a disability from doing what they want are within the power of the local community to put right, for example, access to buildings, suitable transport, suitable housing and the attitudes of people generally towards those with disabilities. The local consultant who is an expert in this field is a valuable resource for these community services ${ }^{4}$ although they may not always realise it at first.

\section{Expanding from clinical neurology into rehabilitation medicine}

I became a consultant neurologist in 1977 and did not really start to practice rehabilitation medicine until 1984. There are three ways in which I have had to change. The first has been to recognise the wider local network of people who need to be involved in an individual disability management plan, and to be prepared to give time and energy to this network. The second set of changes involved the acquisition of new technical clinical skills that are needed to tackle the additional problems effectively. The third change, which is still taking place, and for which I had not been particularly well prepared by my previous experience, is the ability to work in a genuine rehabilitation team and appreciate the nature of the contribution that the other rehabilitation professions and community services have to make.

The technical skills that I have needed (it may not be the same for other neurologists) are first, a better identification and a deeper understanding of the impact of cognitive impairment on a person's ability to adapt, learn new skills, work and maintain social relationships. The second is the capacity to analyse movement in a way that clearly identifies the components that can be helped by medical treatment such as anti-spastic drugs or nerve blocks, by surgery, by orthoses, by better control of posture, by the acquisition of new skills or by the effects of therapy and manipulative procedures. I have had to acquire a smattering of soft-tissue rheumatology and some expertise in orthoses, special seating and wheelchair provision. I have needed to improve my approach to chronic pain problems and to 
those people who believe that they have a physical disability when they do not.

The third new group of skills relates to teamwork. I suspect that many neurologists are a bit like the general practioners in remote rural areas, rather enjoying being on their own and working as individuals. Teamwork takes time and a lot of personal commitment of emotional energy, tolerance and persistence. As an example, our rehabilitation team in Southampton produces a regularly updated and detailed operational policy and an explanatory booklet for patients and families, and has evolved the following timetable of meetings which we find is essential if we are to work effectively:

a) Weekly: 1 Clinical review of progress and objectives.

2 Documentation of current and potential abilities.

3 Education (seminar, tuturial or workshop).

b) Monthly: 1 Business meeting.

2 Audit of the month's discharges.

c) Bi-monthly inter-district audit.

d) Seasonal, and as required, social events.

Neurology or rehabilitation medicine?

From what has been said, it should be clear that neurologists can do what is needed, but it requires a change of orientation and commitment. I have found these changes and this new field stimulating, challenging and of absorbing interest. Clinical neurology would be a more interesting and a more popular discipline if it moved in this direction. There is a risk however. The worst of both worlds would be for neurologists to begin to shift but then only to do half the job, for example, for them to take over the hi-tech aspects of rehabilitation (giving it perhaps another title such as restorative neurology!) while leaving the larger and even more essential components unresourced and undone. To do that would be to develop the service to give satisfaction to traditional neurologists, rather than to provide what people with disabilities and their families require.

\section{Training}

In the UK the current training of senior registrars in neurology is extremely inadequate for these particular needs. I remember talking at a meeting held by the Parkinson's Disease Society in Brighton, and being rendered speechless by this question fired by a member of the audience: "What is to be done about the problem of the cloning of neurologists?" Rehabilitation medicine is at the moment a small specialty with only 24 senior registrars. It would indeed be madness for the UK, which has fewer consultant neurologists and fewer consultants in rehabilitation per head of population than any country in the EEC, to host a competition for territory between these two specialties, both of which are dwarfed by the size of the problems they are expected to overcome. Health districts desperately need medical rehabilitation services and I do not think it matters which specialty a consultant comes from provided that he or she has the commitment, skill, time and determination to learn and to fulfil the ten requirements set out.

\section{Some basic terminology for neurologists}

The contents of a leaflet issued by the New Zealand Employment Service entitled "A few words about disability" is reproduced in full at the end of this paper. This document is included not to demonstrate how far ahead of us New Zealand is in its thinking about approaches to people with disabilities, but because the concepts and, in particular, the terminology has been agreed in all English speaking countries.

\section{APPENDIX \\ "A few words about disability" issued by the New Zealand Employment Service in 1989}

The text of this leaflet, which is distributed free to employers and members of the public by the New Zealand Government Employment Service, is reproduced in full below.

One in every ten people in New Zealand has a disability. This means that the chances are high that you will have contact with at least one person with a disability every day. The disability may not be obvious, and you will probably have no need to mention it. However, if you do need to mention a disability it is important that you use the appropriate language. Your language should reflect a positive, straightfoward, and sensitive attitude towards the person with the disability.

Here are some guidelines and suggestions for preferred language for everyday use. Please consider them when you are talking or writing about people with disabilities.

\section{IMPAIRMENT, DISABILITY OR HANDICAP?}

These words are often used interchangeably. However, they do not mean the same thing. The World Health Organisation has suggested definitions along these lines.

IMPAIRMENT is an anatomical or functional abnormality which may result in disability.

DISABILITY is a loss or reduction of functional ability which results from an impairment.

HANDICAP is the disadvantage caused by the impairment or disability. Handicap represents the social and environmental consequences to the individual stemming from the presence of an impairment or disability.

An example: A 16 year old boy is involved in a traffic accident and his spinal cord is seriously damaged:

impairment - damaged spinal cord

disability - inability to walk

handicap - problems with access to buildings, and finding employment.

\section{SOME GENERAL GUIDELINES}

Do not refer to a person's disability unless it is essential to what you are trying to say about them.

People with disabilities are all individuals and are all different. Avoid labelling them into 
groups such as the disabled, the blind, an epileptic. Instead say people with disabilities, people who are blind, a person with epilepsy.

Where possible emphasise an individual not a disability. Say person or persons with disabilities or a person who is deaf, rather than disabled persons or deaf persons.

Avoid using words which imply pity for people with disabilities such as suffering from, victim of, unfortunate, afflicted with.

Emphasise abilities not disabilities. Say uses a wheelchair rather than cannot walk or confined to a wheelchair, and is partially sighted rather than partially blind.

Most people with disabilities are not sick and should not be referred to as patients unless they are receiving treatment.

Do not perpetuate negative stereotypes by implying that people with disabilities are helpless, bitter, or unattractive. Avoid terms such as invalid, crippled, and deformed.

Do not perpetuate patronising stereotypes by implying that people with disabilities are all courageous in the face of adversity, patient, endowed with special gifts as a compensation for their disability, or childlike.

When describing people without disabilities, non-disabled is the preferred term. Ablebodied is less appropriate because it implies that all people with disabilities have physical disabilities. Normal is not an appropriate alternative to non-disabled.

\section{GUIDELINES ON SPECIFIC DISABILITIES}

The following is a selection of the disabilities which are most often described inappropriately or negatively, and some suggestions for more appropriate and positive terms.

\section{Arthritis}

Rather than saying an arthritic say person with arthritis, person who has arthritis.

\section{Blind}

Describes a person with total loss of vision. Not appropriate for a person with partial vision. Say person who is blind, partially sighted, with partial vision, visually impaired, as appropriate.

\section{Cerebral palsy}

It is not appropriate to describe a person who has cerebral palsy as a spastic. Muscles are spastic not people. Say with cerebral palsy, has cerebral palsy.

\section{Deaf}

Describes a person with total hearing loss. Not appropriate for person with partial hearing. Say person who is deaf, person with partial hearing loss, or hearing impairment, or hearing impaired as appropriate.

Diabetes

Say person who has diabetes, or person with diabetes rather than a diabetic.
Down's Syndrome

The terms Mongol and mongoloid are inappropriate.

\section{Epilepsy}

Rather than saying an epileptic, say person who has epilepsy, or person with epilepsy.

\section{Intellectual disability/handicap}

Intellectual handicap is the term most commonly used in New Zealand but there is growing opinion that intellectual disability is a preferable term.

The term mentally retarded is also sometimes used as a synonym for intellectual disability/handicap, and is commonly used overseas, but it is not appropriate for everyday use in New Zealand. Terms such as, mentally deficient/defective, feeble-minded, idiot, imbecile, are completely inappropriate. Say person with an intellectual disability/handicap, or person who is intellectually disabled/handicapped. Remember also that it is not appropriate to describe an adult with an intellectual disability/handicap as a child.

\section{Mental disability/mental illness}

Definitions and terminology in this area are currently the subject of much debate in New Zealand. There is no clear consensus as to what term is preferable. However, it is clear that terms such as insane, mad, crazy are inappropriate. Terms such as neurotic, psychotic, psychopathic and schizophrenic are specific medical terms and should not be used as general labels in everyday speech and writing.

Depending on context person with a mental disability, or person who has recovered from a mental health problem may be appropriate. Where possible be guided by the person or persons concerned as to what term is preferred.

\section{Person who cannot speak}

Preferred term to describe persons who cannot speak. Terms such as deaf-mute and deaf and dumb are inappropriate. They imply that people without speech are always deaf and vice versa.

\section{REMEMBER THREE THINGS}

People with disabilities are people first. If in doubt ask the person or people concerned what words you should use to refer to their disability. People with disabilities have abilities too.

1 Scottish Home and Health Department. Scottish Health Service Council. Medical rehabilitation: the pattern for the future (Mair Report). Edinburgh:HMSO, 1972.

2 Disability Committee of the Royal College of Physicians of London. Discussion paper, Summary of the nature of the specialty of rehabilitation medicine and projected size of the specialty in England and Wales. London: 1990.

3 McLellan DL. In: M Swash, J Oxbury, eds. Clinical Neurology London: Churchill Livingstone, 1991:785.

4 Hunter J, Walker J. The role of a rehabilitation medicine service. Edinburgh: University of Edinburgh Rehabilitation Studies Unit, 1990.

5 New Zealand Employment Service. $A$ few words about disability. Leaflet: LAB $1086 \mathrm{LMI} 20 \mathrm{M} / 7 / 89$ Wellington: New Zealand Employment Service, 1989. 